\title{
Nanoparticles and the immune system: challenges and opportunities
}

\author{
"...co-delivery of antigen and immune activator to the \\ desired subpopulations of specialized immune cells \\ at the time point when they are most sensitive is an \\ important requirement for a potent activation of the \\ immune system..."
}

First draft submitted: 4 August 2016; Accepted for publication: 19 August 2016; Published online: 20 September 2016

Keywords: cancer immunotherapy • control of immunity • immune tolerance - nanoparticular carriers $\bullet$ removal of immune tolerance

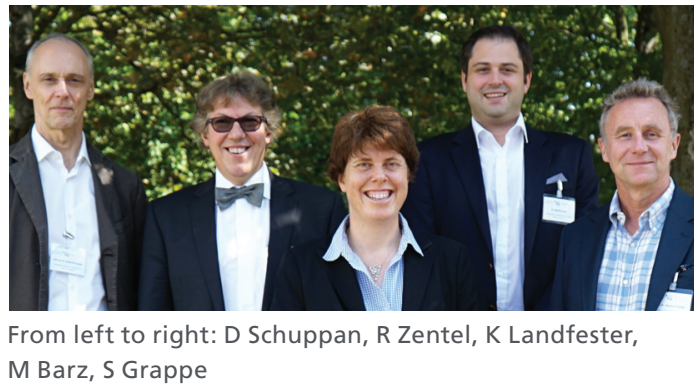

M Barz, S Grappe

The immune system has evolved as our very own defense mechanism to eliminate intracellular or extracellular pathogens from the body. It acts very selectively on the nanoscale and can differentiate precisely between healthy and abnormal situations as well as sense endogenous or exogenous danger signals. In addition, it generates memory, stores information and recalls it later to fight remaining or recurring threats. It is also generally - capable to recognize malignant cells and eliminate metastases in cancer, which are mostly responsible for the failure of standard cancer therapies.

Thus, it has been an attractive approach to treat cancer by targeting the immune system instead of the tumor itself, since fighting tumor directly requires depletion of literally all malignant cells, because even a small number of surviving tumor cells can induce recurrence. Therefore, a traditional anticancer drug needs to reach billions of cells. On the contrary for immunotherapy the activation of several thousand leukocytes is sufficient to induce potent responses, which appears a realistic task [1,2]. These facts provide a striking rationale for employing the immune system to fight cancer leading to first concepts of tumor-immune therapies [3] already at the end of the 19th century.

In recent years, it has been recognized that nonspecific immune activation, for example, by administering immune checkpoint inhibitors, can reactivate natural immunity that apparently evolves spontaneously during the cancer development and progression, and may result in impressive and durable remissions in some cancer entities. However, this type of therapy comes with significant immune-related side effects and only works in a fraction of patients, leaving significant medical need to develop more cancer-specific immunotherapies that are both highly effective and have few side effects. Adoptive transfer of tumor-specific $\mathrm{T}$ cells (e.g., tumorinfiltrating lymphocytes, chimeric antigen receptor-transduced T cells) and tumor vaccination approaches are most promising in this regard. The specific activation of the immune system against a tumor relies on a successful vaccination, meaning that a tumor-associated antigen needs to be presented to the immune system (likely antigen presenting cells) and combined with an immune activator to induce antigen processing and effective induction of T-cell-mediated immunity. This co-delivery of antigen and immune activator
Stephan Grabbe', Katharina Landfester ${ }^{2}$, Detlef

Schuppan ${ }^{3}$, Matthias Barz ${ }^{4}$ \& Rudolf Zentel*,4

'Department of Dermatology, University Medical Center, Johannes GutenbergUniversity Mainz, Obere Zahlbacher Straße 63, 55131 Mainz, Germany ${ }^{2}$ Max-Planck Institute for Polymer Science, Ackermannweg 10, 55128 Mainz, Germany

${ }^{3}$ Institute for Translational Immunology, University Medical Center, Johannes Gutenberg-University Mainz, Langenbeckstrasse 1, 55101 Mainz, Germany

${ }^{4}$ Institute of Organic Chemistry, Johannes Gutenberg-University Mainz, Duesbergweg 10-14, 55099 Mainz, Germany

*Author for correspondence: zentel@uni-mainz.de 
to the desired subpopulations of specialized immune cells at the time point when they are most sensitive is an important requirement for a potent activation of the immune system, since presentation of (tumor) antigen

"...nanoparticles appear especially attractive since they can combine the required functionalities among a single particle...

by an inadequate antigen presenting cell type or in the absence of an immune activator results in immune tolerance rather than immunity [4].

In this respect, nanoparticles appear especially attractive since they can combine the required functionalities among a single particle being in the same size range $(\mathrm{nm})$ as viruses and fragments of bacteria, for which our immune system evolved naturally.

In addition, nanoparticles can effectively protect and shield biologically sensitive molecules (e.g., antigen-bearing peptides, DNA or mRNA encoding for antigens or immunostimulatory oligonucleotides) from degradation, increase their half-life in the body and minimize their systemic toxicity [5-7]. This enables novel therapeutic approaches, which provide - besides

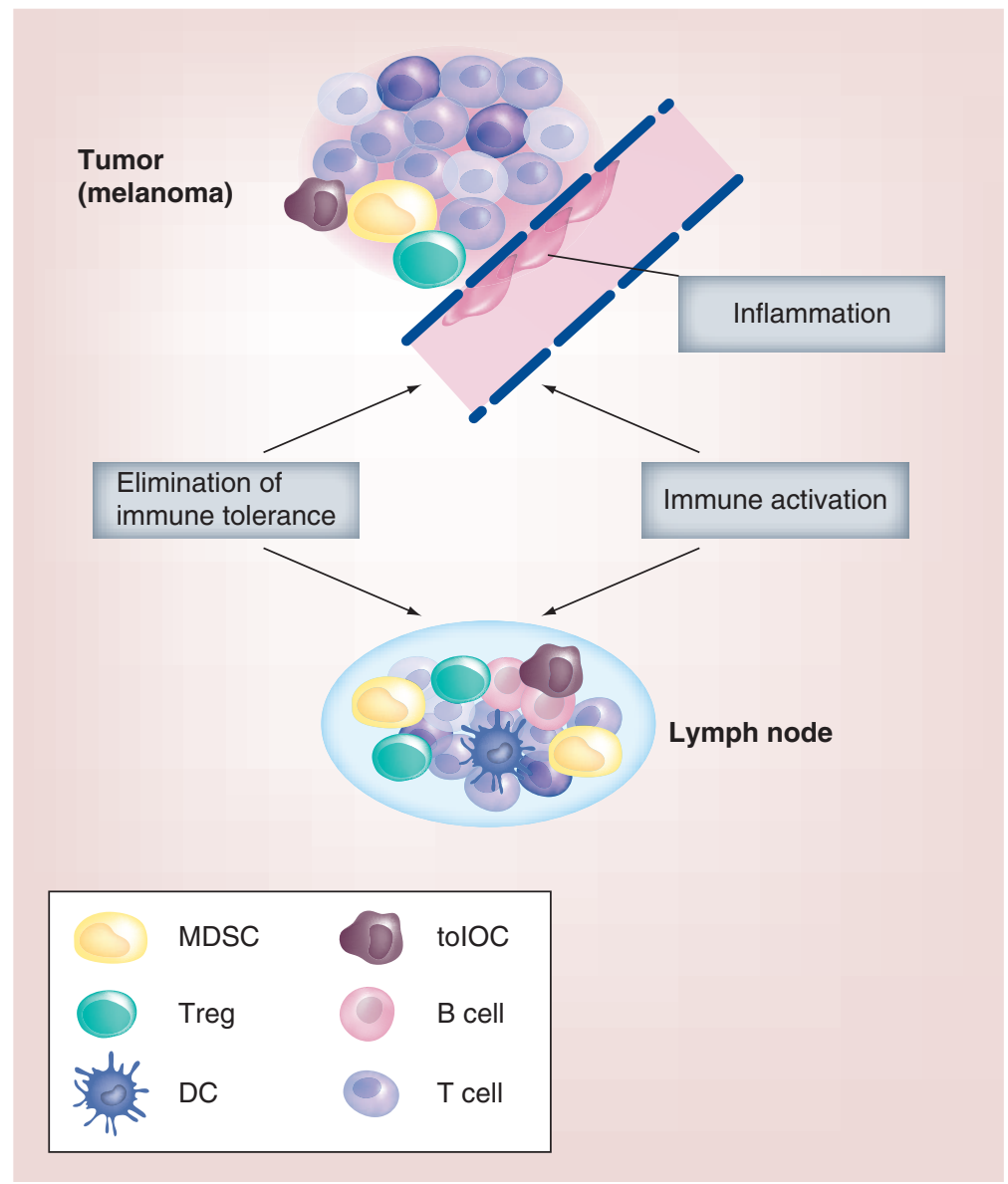

Figure 1. Attacking the tumor by combining immune activation, elimination of immune tolerance and induction of an inflammation. local applications - the possibility for systemic activation of the immune system. In this context, a lot can be learned from efforts to optimize nanoparticles for tumor treatment [8], which requires also prolonged circulation and specific delivery. Interestingly, some sortsof 'passive' accumulation of nanoparticles also exists within the immune system. It has been recently demonstrated that after intradermal injection nanoparticles accumulate in the lymph nodes [9], whenever the interstitial flow is able to transport them through the lymphatic capillaries into the draining lymph nodes, where these particles can be taken up by antigenpresenting immune cells without further targeting ligands $[10,11]$. Second, depending on size and surface charge, nanoparticles may selectively accumulate in certain organs such as spleen or liver, which can be beneficial for systemic immune therapies since these organs have micromilieus that favor the generation of immunity or tolerance, respectively.

For both cases the material of the nanocarrier is of major importance. Unspecific immune activation or major nanocarrier aggregation in the blood needs to be avoided by any applied carrier material. Thus, nanocarriers need to be carefully evaluated preclinically for biodegradability, immune-mediated and nonimmunemediated toxicity, stability and ability to be produced in a standardized, reproducible fashion according to good manufacturing practice requirements.

The successful development of materials and carrier systems thereof can only be performed in an interdisciplinary and directed manner. Our collaborative research center for nanoparticle-based cancer immune therapy (CRC 1066) exactly provides these desired properties [12-14]. Here especially physicochemical characterization techniques provide insights into the behavior of nanocarriers in complex media, like blood or intracellular fluids [15,16]. In such a complex mixture of proteins it turned out that the formation of a protein corona is likely to occur for many nanoparticles and its composition relates to the material used to construct the carrier systems and thus cannot be disregarded whenever the behavior of the whole system is investigated [16]. Nevertheless, a successful targeting of immune cells seems possible [17,18]. To that respect, the potential of nanoparticles for immunotherapy in general - is enormous $[19,20]$ or, in other words 'The immune system likes nanotechnology' [21,22].

However, while vaccination against infectious diseases has revolutionized human healthcare in the last century, it is still difficult to transfer this strategy to diseases derived from the body's own tissue as in cancer. In this case, malignant cells develop mechanisms to escape from efficient immune-mediated eradication. Acquired immune resistance limits then - not only - natural anti- 
tumor immune responses but also represents a major barrier to efficiently treat the disease. Cancer immunotherapy encompasses a variety of approaches that aims to re-engage the immune system to seek and destroy cancer cells. Obstacles to this, are, for example, particular tumor-cell properties and immune system components such as activated regulatory $\mathrm{T}$ cells [23] and myeloid-derived suppressor cells. However, to avoid severe adverse effects, these cell types and structures need to be specifically therapeutically targeted.

Here, nanoparticles provide a new means in sitespecific drug delivery and functional alteration of cells and thus hold substantial potential to improve treatment of cancer. First success to break tumor immune-tolerance could recently be made by blocking immunosuppressive receptors (e.g., CLT4-A or PD-1) with monoclonal antibodies abrogating their tumor promoting potential on $T$ cells targeted against the tumor [3] or low molecular weight drugs interfering with intratumoral cyclic adenosine monophosphate levels [23] as recently demonstrated within our consortium.

However, to combat the tumor successfully a combination of antigen-specific and nonantigen-specific immune activation, the elimination of immune tolerance and the induction of an inflammation within the tumor to recruit immune cells seems to be a necessity (Figure 1). Therefore, the collaborative research center for nanoparticle-based cancer immune therapy (CRC 1066) started with the vision to combine all three aspects to develop potent cancer immune therapies. Now 3 years later, first results in mice and men clearly point in this direction. The group of Ugur Sahin, member of the CRC 1066, has recently gener-

\section{References}

1 Banchereau J, Steinman RM. Dendritic cells and the control of immunity. Nature 392, 245-252 (1998).

2 Tacken PJ, de Vries IJ, Torensma R, Figdor CG. Dendriticcell immunotherapy: from ex vivo loading to in vivo targeting. Nat. Rev. Immunol. 7, 790-802 (2007).

3 Dömling A, Holak TA. Programmed death-1: therapeutic success after more than 100 years of cancer immunotherapy. Angew. Chem. Int. Ed. Engl. 53(9), 2286-2288 (2014).

4 Van Parijs L, Abbas AK. Homeostasis and self-tolerance in the immune system: turning lymphocytes off, Science 280 (5361), 243-248 (1998).

5 Sahin U, Karikó K, Türeci Ö. mRNA-based therapeuticsdeveloping a new class of drugs, Nat. Rev. Drug Discov. 13(10), 759-780 (2014).

6 Hartmann S, Nuhn L, Palitzsch B et al. CpG-loaded multifunctional cationic nanohydrogel particles as self- ated mRNA containing lipoplexes that potently target and activate dendritic cells in the spleen, resulting in potent antitumor immune responses in rodents as well as in patients [7], while they reduce tumor tolerance at the same time. Future work in this direction has to be made in close cooperation between materials science and biomedical research. It requires well-defined, biocompatible and - probably - stimuli responsive carriers, the careful characterization of them in relevant body fluids and detailed immunological evaluation, which we brought together in the established center.

\section{6}

...to combat the tumor successfully a combination of ... immune activation, the elimination of immune tolerance and the induction of an inflammation within the tumor to recruit immune cells seems to be a necessity.

This special issue of nanomedicine combines various aspects necessary for nanoparticle-based cancer immune therapy, from intracellular trafficking, via nanocarriers and their interaction with the players of the immune system to imaging and translation. Special attention is thereby put on the aspect of immune tolerance, which has been - so far - often overlooked in this context.

\section{Financial \& competing interests disclosure}

The authors have no relevant affiliations or financial involvement with any organization or entity with a financial interest in or financial conflict with the subject matter or materials discussed in the manuscript. This includes employment, consultancies, honoraria, stock ownership or options, expert testimony, grants or patents received or pending, or royalties.

No writing assistance was utilized in the production of this manuscript.

adjuvanting glycopeptide antitumor vaccines. Adv. Healthc. Mater. 4, 522-527 (2015).

7 Kranz LM, Diken M, Haas H et al. Systemic RNA delivery to dendritic cells exploits antiviral defence for cancer immunotherapy. Nature 534(7607), 396-401 (2016).

8 Lammers T, Kiessling F, Hennink WE, Storm G. Drug targeting to tumors: principles, pitfalls and (pre-) clinical progress. J. Control. Release 161, 175-187 (2012).

9 Reddy ST, Rehor A, Schmoekel HG, Hubbell JA, Swartz MA. In vivo targeting of dendritic cells in lymph nodes with poly(propylene sulfide) nanoparticles. J. Control. Release 112(1), 26-34 (2006).

10 Reddy ST, van der Vlies AJ, Simeoni E, O'Neil CP, Swartz MA, Hubbell JA. Exploiting lymphatic transport and complement activation in nanoparticle vaccines. Nat. Biotechnol. 25(10), 1159-1164 (2007).

11 Nuhn L, Vanparijs N, De Beuckelaer A et al. pH-Degradable imidazoquinoline-ligated nanogels for lymph node focused immune activation. Proc. Natl Acad. Sci. USA 113(29), 8098-8103 (2016). 
12 Nuhn L, Barz M, Zentel R. New perspectives of HPMAbased copolymers derived by post-polymerization modification (feature). Macromol. Biosci. 14, 607-618 (2014).

13 Klinker K, Barz M. Polypept(o) ides: hybrid systems based on polypeptides and polypeptoids (feature). Macromol. Rapid Comm. 36, 1943-1957 (2015).

14 Kang B, Opatz T, Landfester K, Wurm F. Carbohydrate nanocarriers in biomedical applications: functionalization and construction. Chem. Soc. Rev. 44, 8301-8325 (2015).

15 Fischer K, Schmidt M. Pitfalls and novel applications of particle sizing by dynamic light scattering. Biomaterials 98 , 79-91 (2016).

16 Schoettler S, Becker G, Winzen S et al. Protein adsorption is required for stealth effect of poly(ethylene glycol)- and poly(phosphoester)-coated nanocarriers. Nat. Nanotech. 11, 372-377 (2016).

17 Kang B, Okwieka P, Schoettler S et al. Carbohydrate-based nanocarriers exhibiting specific cell targeting with minimum influence from the protein corona. Angew. Chem. Int. Ed. Engl. 54, 7436-7440 (2015).
18 Tappertzhofen K, Bednarczyk M, Koynov K, Bros M, Grabbe S, Zentel R. Towards anticancer immunotherapeutics: well-defined polymer-antibody conjugates for selective dendritic cell targeting. Macromol. Biosci. 14, 1444-1457 (2014).

19 Shao K, Singha S, Clemente-Casares X, Tsai S, Yang Y, Santamaria P. Nanoparticle-based immunotherapy for cancer. ACS Nano 9(1), 16-30 (2015).

20 Irvine DJ, Hanson MC, Rakhra K, Tokatlian T. Synthetic nanoparticles for vaccines and immunotherapy. Chem. Rev. 115, 11109-11146 (2015).

21 Lepeltier E, Nuhn L, Lehr CM, Zentel R. Not just for tumor targeting. Unmet medical needs and opportunities for nanomedicine. Nanomedicine 10(20), 3147-3166 (2015).

22 Toth I, Skwarczynski M. The immune system likes nanotechnology. Nanomedicine (Lond.). 9(17), 2607-2609 (2014).

23 Bacher N, Raker V, Hofmann C et al. Interferon- $\alpha$ suppresses cAMP to disarm human regulatory $\mathrm{T}$ cells. Cancer Res. 73(18), 5647-5656 (2013). 